\title{
Inhibition of angiogenesis by platelets in systemic sclerosis patients
}

\author{
Daniela Hirigoyen ${ }^{1}$, Paula I. Burgos', Veronica Mezzano', Josefina Duran', Magaly Barrientos ${ }^{1}$, Claudia G. Saez²,
} Olga Panes ${ }^{2}$, Diego Mezzano ${ }^{2}$ and Mirentxu Iruretagoyena ${ }^{1^{*}}$

\begin{abstract}
Introduction: Systemic sclerosis (SSC) is a chronic autoimmune disease characterized by microvascular damage, inflammation, and fibrosis. It has become increasingly evident that platelets, beyond regulating hemostasis, are important in inflammation and innate immunity. Platelets may be an important source of proinflammatory and profibrotic cytokines in the vascular microenvironment. In this study, we sought to assess the contribution of platelet-derived factors in patients with SSC to the angiogenesis of human dermal microvascular endothelial cells (DMVECs) in a tubule formation assay and to characterize the secretion of profibrotic and proinflammatory cytokines in these platelets.
\end{abstract}

Methods: We analyzed platelets obtained from 30 patients with SSC and 12 healthy control subjects. Angiogenesis was evaluated in vitro with a DMVEC tubule formation assay on Matrigel and platelet-derived angiogenic factors such as vascular endothelial growth factor (VEGF), 165b isoform (VEGF $165 \mathrm{~b})$, and cytokine secretion was evaluated. Platelet serotonin content was also determined.

Results: When DMVECS were incubated with SSc platelet releasates, tubule formation was significantly inhibited $(p<0.01, t$ test), and higher expression of endothelin- 1 in these cells was observed compared with control subjects $(p<0.05$, Mann-Whitney $U$ test). In SSc platelet releasates, VEGF 165 bas significantly higher $(p<0.05, t$ test), and the VEGF ${ }_{165} \mathrm{~b} / \mathrm{NEGF}$ ratio was increased compared with that of control subjects. Higher secretion of transforming growth factor $\beta$ ( $p<0.01, t$ test) and CD40L ( $p<0.01, t$ test) was observed compared with control subjects. Also, intraplatelet serotonin levels were lower in platelets obtained from patients with diffuse SSc compared with patients with limited SSC and control subjects ( $p<0.05, t$ test).

Conclusions: Our findings suggest that antiangiogenic factors such as $\mathrm{VEGF}_{165} \mathrm{~b}$, together with proinflammatory and profibrotic factors secreted by platelets, can contribute to the progression of peripheral microvascular damage, defective vascular repair, and fibrosis in patients with SSC.

Keywords: Systemic sclerosis, Platelets, Vascular endothelial growth factor, Transforming growth factor $\beta$

\section{Introduction}

Systemic sclerosis (SSc), or scleroderma, is a chronic autoimmune disease characterized by vasculopathy, immune deregulation, and fibrosis of the skin and internal organs. It has an estimated prevalence of about 1-2 per 100,000 persons [1]. Women are at much higher risk for developing scleroderma, generally starting with symptoms in middle age [2]. Clinically, the disease is classified into two major

\footnotetext{
*Correspondence: mitureta@med.puc.cl

'Departamento de Inmunología Clínica y Reumatología, Pontificia Universidad Católica de Chile, Marcoleta 350, Santiago, Chile Full list of author information is available at the end of the article
}

subgroups according to the extension of skin involvement (limited or diffuse cutaneous scleroderma) and the autoantibody profile. Both types can present with vascular involvement, Raynaud's phenomenon and obliterative vasculopathy, and systemic fibrosis [3]. The pathogenic triad involving vascular injury, autoimmunity, and fibrosis (scleroderma trifecta) is classically considered a hallmark of the disease, but the interplay among these distinct processes, which initiate and sustain the progressive tissue damage in scleroderma, requires further exploration [4-6]. Both autoimmunity and vasculopathy appear to precede the onset of the disease, and contribute to the progression of fibrosis. 
Platelets play key roles in hemostasis, thrombosis, and tissue repair and, as more recently described, in inflammation and innate immunity [7]. Platelets are currently being used, among others, for regenerative treatments after bone surgery and cosmetic surgery, although solid evidence of their regulatory functions is missing. Endothelial dysfunction and immune regulatory mechanisms may be associated with platelet function. It has been proposed that platelets can play a role in SSc because enhanced activation and an increased tendency to aggregation have been described in these patients [8].

Platelets could substantially contribute to the pathophysiology of scleroderma through several mechanisms. The levels of several platelet-derived molecules, such as $\beta$-thrombomodulin and platelet-derived growth factor, are elevated in the serum of patients with SSc $[8,9]$. In addition, elevated levels of circulating platelet aggregates have been described [8]. Silveri et al. found higher levels of endothelium-derived endothelin-1, tissue type plasminogen activator, plasminogen activator inhibitor type 1 , and platelet-derived growth factor in patients with Raynaud's phenomenon associated with scleroderma than in control subjects, suggesting platelet activation in vivo and endothelial damage [10]. However, the exact contribution of platelets and platelet-derived factors in the pathogenesis of SSc and other fibrotic diseases has not yet been established.

Our hypothesis is that platelets contribute to maintaining the microvascular injury that leads to fibrosis in patients with SSc. The aim of this study was to further characterize the role of platelets in vascular damage in patients with scleroderma and their proinflammatory and profibrotic cytokine profiles. In particular, the angiogenesis of human dermal microvascular endothelial cells (DMVECs) was evaluated in the presence of platelet supernatants obtained from patients with SSc. Also, antiangiogenic factors such as vascular endothelial growth factor (VEGF), 165b isoform $\left(\mathrm{VEGF}_{165} \mathrm{~b}\right)$, were assessed. Serotonin content, cytokine secretion profile, and fibroblast proliferation and stimulation by SSc and control platelets were also evaluated. Our results add further evidence for the participation of platelets in microvascular damage and fibrosis induction in SSc.

\section{Material and methods}

\section{Patients}

We studied platelets derived from 30 patients with SSc, including both women and men (mean 51.3 years), and from 12 healthy control subjects. The diagnosis of SSc was made according to the American College of Rheumatology criteria [11], and the patients were classified into diffuse and limited types according to the criteria described by Le Roy et al. [12]. The clinical characteristics of the patients are summarized in Table 1. All samples were
Table 1 Demographic and clinical characteristics of patients with SSc and control subjects

\begin{tabular}{|c|c|c|}
\hline Characteristic & Patients $(n=30)$ & $\begin{array}{l}\text { Control subjects } \\
(n=12)\end{array}$ \\
\hline Female sex, \% & 96.6 & 91.6 \\
\hline Age, yr, mean (SD) & $51.3(16.8)$ & $41.9(14.7)$ \\
\hline Disease duration, mo, mean (SD) & $128.5(18.4)$ & \\
\hline \multicolumn{3}{|l|}{ Disease subset } \\
\hline Diffuse systemic sclerosis, \% & 17.2 & \\
\hline Limited systemic sclerosis, \% & 82.8 & \\
\hline ANA positivity $(>1: 80), \%$ & 100 & 0 \\
\hline Centromere, \% & 71.4 & \\
\hline Nucleolar, \% & 3.7 & \\
\hline Other, \% & 25 & \\
\hline Anti-SCl-70 & 16.1 & \\
\hline Anti-RNA polymerase III & 6.7 & \\
\hline $\mathrm{ESR}, \mathrm{mm} / \mathrm{h}$, mean (SD) & 32.7 (33.6) & \\
\hline Hemoglobin, g/dl, mean (SD) & $12.5(1.2)$ & \\
\hline 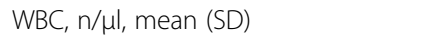 & $7288.21(2836.6)$ & \\
\hline Platelets, $10^{9} / \mathrm{L}$, mean (SD) & $245(65)$ & \\
\hline CRP, mg/L, mean (SD) & $0.47(0.5)$ & \\
\hline Ultrasensitive CRP $\mu \mathrm{g} / \mathrm{ml}$, mean (SD) & $12.24(28.2)$ & $5.08(1.0)$ \\
\hline $\begin{array}{l}\text { Complement component } \mathrm{C} 3, \mathrm{mg} / \mathrm{dl} \text {, } \\
\text { mean (SD) }\end{array}$ & $104.42(20.2)$ & \\
\hline $\begin{array}{l}\text { Complement component } \mathrm{C} 4, \mathrm{mg} / \mathrm{dl} \text {, } \\
\text { mean (SD) }\end{array}$ & $17.42(5.79)$ & \\
\hline Raynaud's phenomenon, \% & 89.66 & \\
\hline Gastrointestinal symptoms, \% & 75.0 & \\
\hline Digital ulcers, \% & 31.3 & \\
\hline Calcinosis, \% & 17.2 & \\
\hline Lung fibrosis, \% & 23.3 & \\
\hline Pulmonary arterial hypertension, $\%$ & 13.3 & \\
\hline \multicolumn{3}{|l|}{ Current therapies, \% } \\
\hline Mycophenolate mofetil & 16.7 & \\
\hline Methotrexate & 26.7 & \\
\hline Cyclophosphamide & 6.7 & \\
\hline Prednisone & 18.7 & \\
\hline
\end{tabular}

ANA antinuclear antibodies, CRP C-reactive protein, ESR erythrocyte sedimentation rate, IQR interquartile range, $S D$ standard deviation, $W B C$ white blood cell count

obtained after the patients and control subjects gave their written informed consent in accordance with the Declaration of Helsinki. The ethics committees of the Pontificia Universidad Católica de Chile and Comisión Nacional de Investigación Científica y Tecnológica (CONICYT) approved the entire study protocol. Patients and control subjects taking aspirin or serotonin reuptake inhibitors were excluded from the study. 


\section{Platelet isolation}

Platelets were prepared as previously described [13]. Briefly, venous blood $(40 \mathrm{ml})$ was collected from patients and control subjects and diluted in acid citrate dextrose formula A solution (1:10 vol/vol). After centrifugation $(10$ minutes at $150 \times g)$ the platelet-rich plasma was collected and centrifuged $(10$ minutes at $150 \times g)$. The pellet was washed with Tyrode's solution (137 mM NaCl, $5.3 \mathrm{mM} \mathrm{KCl}, 1 \mathrm{mM} \mathrm{MgCl} 2,2 \mathrm{mM} \mathrm{CaCl}_{2}, 4.1 \mathrm{mM}$ $\mathrm{NaCO}_{3}$, and $5.5 \mathrm{mM}$ glucose, $\mathrm{pH}$ 6.5, containing 120 $\mathrm{nM}$ prostaglandin $\mathrm{E}_{1}$ ). Platelets were centrifuged to remove residual leukocytes and finally resuspended in the same $\mathrm{Ca}^{2+}$-free buffer. Leukocyte contamination was evaluated by fluorescence microscopy using propidium iodide staining. Leukocyte counts were always less than $1 / 10^{6}$ platelets. In addition, monocyte contamination was evaluated by amplification of CD14 messenger RNA (mRNA) in polymerase chain reactions (PCRs). Isolated, washed platelets at $600,000 / \mu \mathrm{l}$ were incubated in the presence or absence of $2 \mu \mathrm{g} / \mathrm{ml}$ equine collagen (collagen reagent; Hormon-Chemie, Munich, Germany) for 5 minutes at $37{ }^{\circ} \mathrm{C}$ in a four-channel PAP-4 aggregometer (Bio/Data, Horsham, PA, USA). The slope and maximal platelet aggregation were recorded for 5 minutes. Then platelet suspensions were centrifuged at $10,000 \times g$ for 5 minutes, and supernatants were collected and stored at $-80{ }^{\circ} \mathrm{C}$ until processing.

\section{Measurement of inflammatory mediators}

Transforming growth factor $\beta$ (TGF- $\beta$ ), CD40L, tumor necrosis factor (TNF)- $\alpha$, VEGF, and VEGF $_{165}$ b were measured in platelet supernatants by using commercial quantitative colorimetric sandwich enzyme-linked immunosorbent assays (ELISAs) (catalogue numbers DB100B, DCDL40, DTA00C, DVE00, and DY3045, respectively; R\&D Systems, Minneapolis, MN, USA) and connective tissue growth factor (CTGF) was measured in platelet supernatants by using another ELISA (catalogue number RHF461CKX; Antigenix America, Huntington Station, NY, USA) according to the manufacturers' instructions. Concentrations were calculated using a standard curve generated with specific standards provided by the manufacturers. Samples for TGF- $\beta$ analysis were acid-activated with $1 \mathrm{~N} \mathrm{HCl}$. Optical density was measured with a microtiter plate reader at $450 \mathrm{~nm}$. Each sample was measured in triplicate. von Willebrand factor (VWF) was measured in serum by ELISA as described previously [14].

\section{Intraplatelet serotonin determination}

Intraplatelet serotonin (5-hydroxytryptamine) was measured using a high-performance liquid chromatography (HPLC) technique [15]. The HPLC system consisted of Ultrasphere 5- $\mu \mathrm{m}$ ODS column, $250 \times 4.6 \mathrm{~mm}$ (HiChrom, Theale, UK), a Waters 515 HPLC pump (Waters, Milford,
MA, USA), a Rheodyne manual injector (Sigma-Aldrich, St. Louis, MO, USA), an electrochemical detector (Waters 464), and EMPOWER software (Waters). A platelet sample $(20 \mu \mathrm{l})$ was injected for HPLC analysis, and the amount of serotonin was calculated on the basis of a calibration curve.

\section{Angiogenesis and fibroblast proliferation assays}

To assess platelet-derived angiogenic and antiangiogenic factors, in vitro tubule formation assays were performed with human DMVECs [catalogue number CRL-4025; American Type Culture Collection (ATCC), Manassas, VA, USA] cultured in 24-well plates covered with Matrigel culture mix (BD Biosciences, San Diego, CA, USA). Briefly, Matrigel $(200 \mu \mathrm{l})$ was pipetted into culture wells and polymerized for 30 minutes at $37{ }^{\circ} \mathrm{C}$. Then DMVECs that formed a small number of short tubular structures when cultured alone were cultured in duplicate in endothelial growth basal medium (EBM-2) supplemented with EBM-2MV SingleQuots (Lonza, Walkersville, MD, USA) on 24-well plates. Each experiment was conducted by pairing samples of platelet supernatants (10\% $\mathrm{vol} / \mathrm{vol}$ ) derived from patients with SSc and from healthy control subjects. As a control, DMVECs were supplemented with VEGF $(10 \mathrm{ng} / \mathrm{ml})$ or cultured alone on Matrigel. Cells were photographed at $6 \mathrm{~h}$. The results were quantified by measuring the total tube length in each well.

To assess platelet-derived TGF- $\beta$, human lung fibroblasts (WI-38, catalogue number CCL-75; ATCC) were cultured in 24-well plates in Dulbecco's modified Eagle's medium (DMEM) supplemented with $10 \%$ fetal bovine serum. For proliferation assays, fibroblasts were cultured with $10 \% \mathrm{vol} / \mathrm{vol} \mathrm{SSc}$ or control platelet supernatant and after $36 \mathrm{~h}$ and proliferation was assessed with the CellTiter 96 Non-Radioactive Cell Proliferation Assay [3-(4,5-dimethylthiazol-2-yl)-2,5-diphenyltetrazolium bromide (MTT); Promega, Madison, WI, USA) according to the manufacturer's instructions. Briefly, after $36 \mathrm{~h}$ in fibroblast culture, MTT was added to the cells, followed by incubation at $37{ }^{\circ} \mathrm{C}$ for $4 \mathrm{~h}$. Then each well was incubated for $1 \mathrm{~h}$ with solubilization stop solution, and absorbance was analyzed using an ELISA reader at $570 \mathrm{~nm}$. Untreated fibroblasts were used as a negative control. The results were normalized to untreated cells. Fibroblasts incubated with $5 \mathrm{ng} / \mathrm{ml}$ TGF- $\beta$ were used as a positive control.

\section{Quantitative real-time RT-PCR of endothelin-1 and a-smooth muscle actin}

Gene expression was quantified by performing TaqMan real-time reverse transcription (RT)-PCR with a PRISM 7500 System (Applied Biosystems, Foster City, CA, USA). Total RNA was isolated from DMVECs and fibroblasts with TRIzol reagent (Life Technologies, Grand Island, NY, USA). RT into complementary DNA (cDNA) was performed using avian myeloblastosis virus reverse transcriptase (Promega), 
with random primers. A predeveloped RPL32 TaqMan gene expression assay (Hs00851655-g1; Life Technologies) was used to normalize for the amounts of loaded cDNA. Differences were calculated with the comparative cycle threshold method for relative quantification. Endothelin-1 (Hs00174961-m1; Life Technologies) and $\alpha$-smooth muscle actin ( $\alpha$-SMA) (Hs00426835-g1; Life Technologies) gene expression was evaluated with TaqMan assays.

\section{Statistical analyses}

Statistical analyses were performed using SAS version 9.1 software (SAS Institute, Cary, NC, USA). Data are shown as mean \pm standard error of the mean or median and range. A two-sided non-parametric Mann-Whitney $U$ test was used for independent samples, and Student's $t$ test was performed for paired samples. Analysis of variance (ANOVA) was used for multiple comparisons. $p<0.05$ was considered statistically significant.

\section{Results}

DMVEC angiogenesis is inhibited by SSc platelet releasates Tissue fibrosis is preceded by microvascular injury in SSc, leading to a progressive loss of capillaries [16]. The effect of platelet releasates in angiogenesis was studied using a tubule formation assay with DMVECs cultured in Matrigel for $6 \mathrm{~h}$. The capacity of platelet releasates to enhance tubular formation was expressed as the total tube length in the culture. Figure $1 \mathrm{a}-\mathrm{b}$ shows that the conditioned medium from SSc platelets significantly inhibited tubule formation in Matrigel cultures compared with supernatants from healthy control platelets. The graph shows the results of six different duplicate experiments $(p<0.01, t$ test). We found no differences according to disease subset (data not shown).

We also evaluated endothelin-1 mRNA expression in DMVECs after $24 \mathrm{~h}$ of culture in medium (control) or platelet releasates from healthy control subjects and patients with SSc. Endothelin-1 was upregulated after culturing the cells for $6 \mathrm{~h}$ with SSc platelet supernatant (Fig. 1c) $(p<0.05$, Mann-Whitney $U$ test for healthy control vs. SSc platelet releasates). Moreover, the supernatants from healthy control platelets reduced the endothelin-1 mRNA expression compared with non-stimulated DMVECs.

Several studies have shown that VEGF expression is markedly increased in the epidermis and dermis of patients with SSc $[17,18]$. As recently reported [19], there

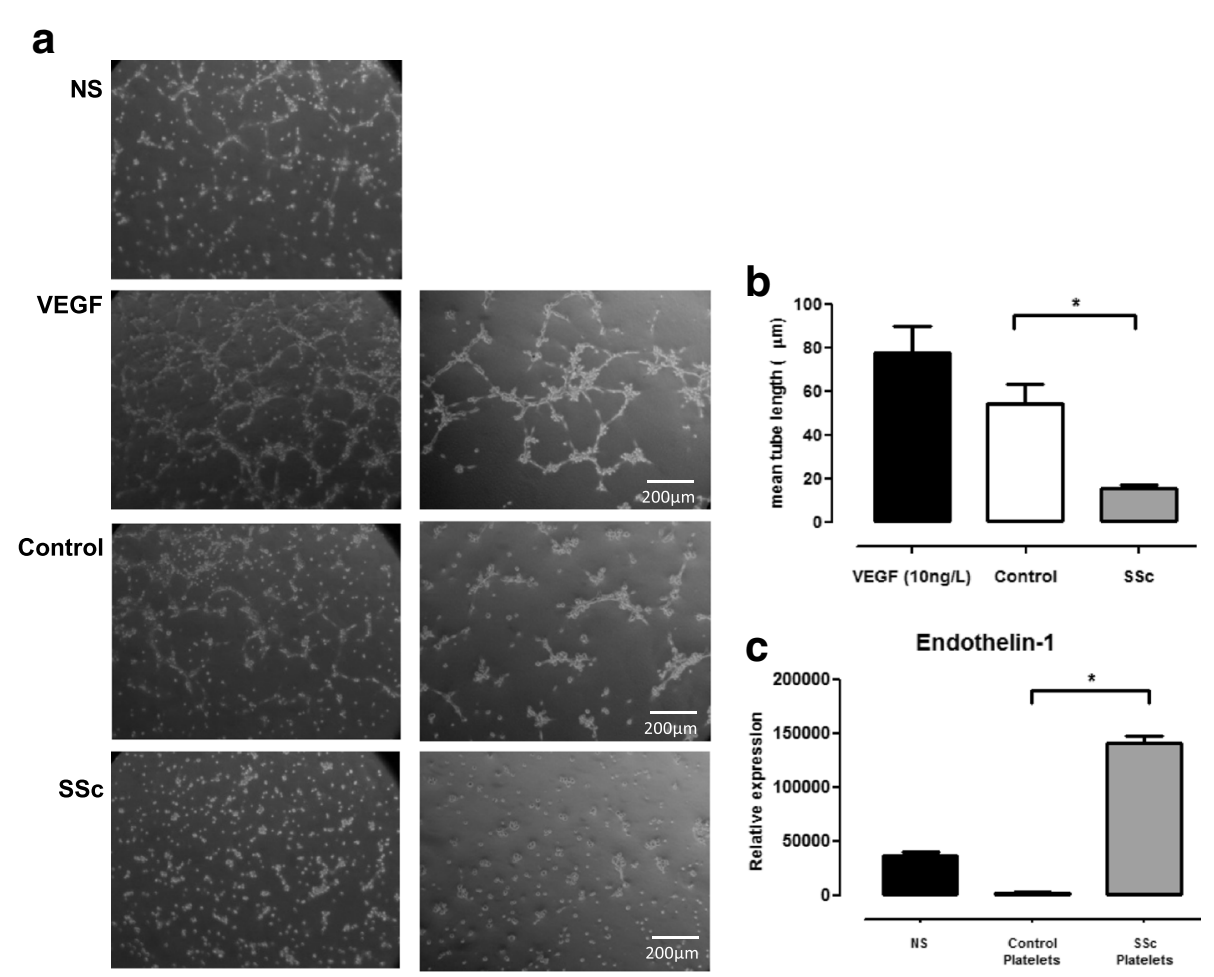

Fig. 1 Systemic sclerosis (SSc) platelet releasate inhibits angiogenesis. a Representative images of Matrigel cultures of dermal human microvascular endothelial cells (DMVECs) after $6 \mathrm{~h}$ in culture. Healthy control and SSc platelet supernatants were cultured at $10 \%$ vol/vol. Left and right columns represent different magnifications (magnification is 10x for the first column and 20x for the second column). $\mathbf{b}$ Capillary morphogenesis was quantified by measuring the length of capillary projections ( ${ }^{*} p<0.01, t$ test). Data are mean \pm standard deviation of six duplicate experiments. $\mathbf{c}$ Endothelin-1 mRNA expression in DMVECs after $24 \mathrm{~h}$ of culture in platelet-conditioned medium. Endothelin-1 was upregulated after the cells were cultured with SSc platelet supernatants ( $p<0.05$, Mann-Whitney $U$ test). NS non-stimulated, VEGF vascular endothelial growth factor 
are splice variants of VEGF with antiangiogenic effects, one of them being $V_{E G F}{ }_{165} \mathrm{~b}$. Increased $V_{E G F}{ }_{165} \mathrm{~b}$ has been shown in serum and in skin biopsies of patients with SSc [20]. Given that platelets contain high amounts of VEGF, we measured the levels of VEGF ${ }_{165} \mathrm{~b}$ in platelet supernatants. As shown in Fig. 2a, SSc platelet supernatants contained significantly higher levels of $\mathrm{VEGF}_{165} \mathrm{~b}$ than healthy control platelet supernatants $(n=12 ; p<0.05, t$ test). The ratio of antiangiogenic VEGF $_{165} \mathrm{~b}$ to total VEGF in platelet supernatants was higher in patients with SSc than in control subjects, but the difference did not reach statistical significance ( $n=12 ; p=0.071, t$ test) (Fig. 2b). Taken together, these results support an antiangiogenic role of $\mathrm{VEGF}_{165} \mathrm{~b}$ in microvascular damage.

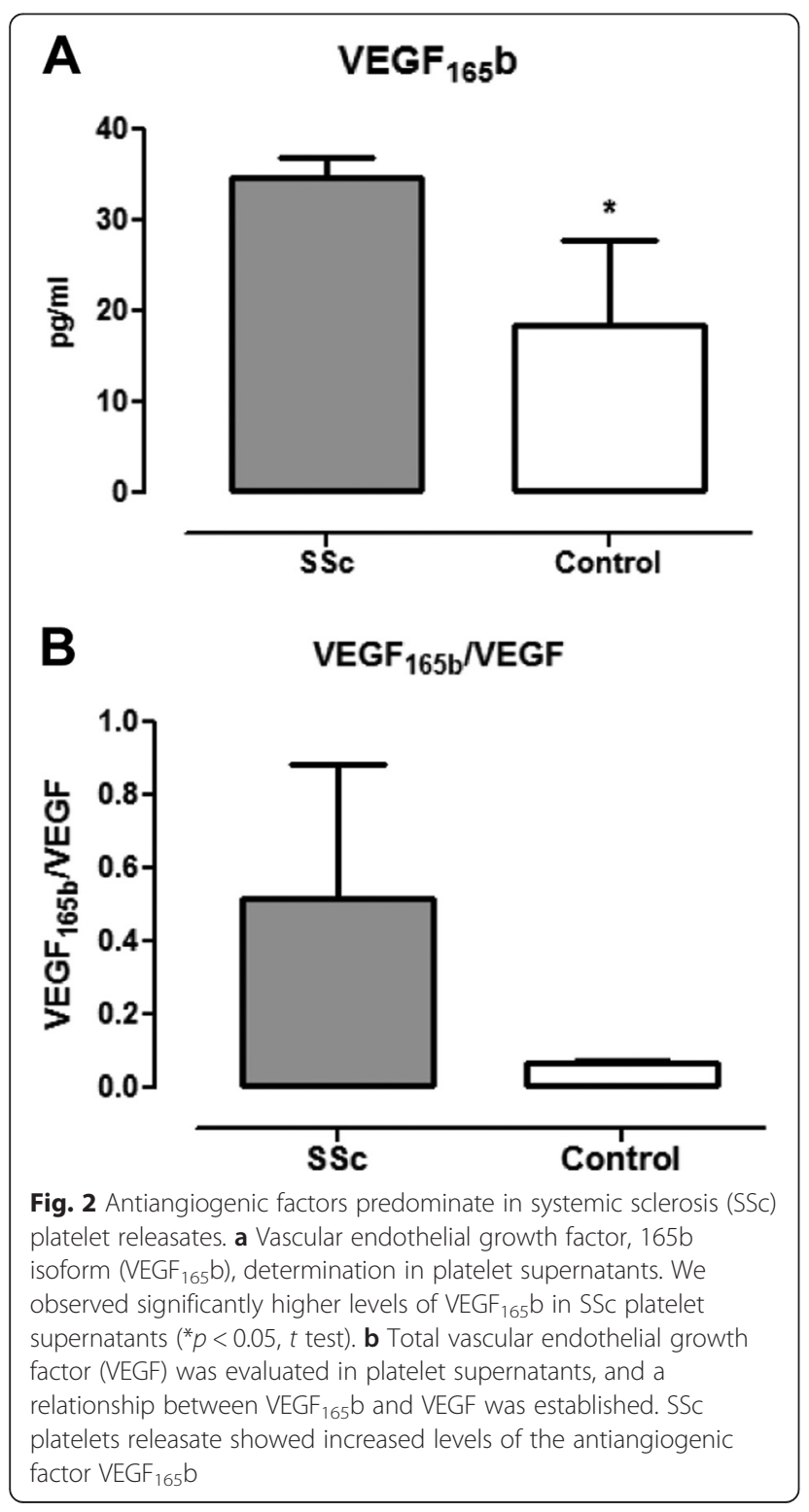

\section{Proinflammatory and profibrotic cytokines secreted by SSc-derived platelets}

The releasates (basal and postcollagen) obtained after platelet isolation were analyzed for TGF- $\beta$, TNF- $\alpha$, CTGF, and CD40L levels (Fig. 3a, b). We observed significant increases in basal TGF- $\beta(p<0.01, t$ test $)$ and CD40L $(p<$ $0.01, t$ test) secretion in platelets from patients with SSc compared with those from healthy control subjects. After collagen stimulation, platelets from patients with SSc also secreted higher levels of TGF- $\beta$ and CD40L, although the differences were non-significant in this experimental setting ( $p=0.07$ for TGF- $\beta$ and $p=0.05$ for CD40L, $t$ tests). The slight increases of TNF- $\alpha$ and CTGF in basal and activated supernatants of SSc platelets compared with supernatants of control platelets were not statistically significant.

It has been suggested that platelet-derived serotonin can stimulate extracellular matrix synthesis in interstitial fibroblasts [21]. This observation led us to measure intraplatelet serotonin content in patients with SSc. As observed in Fig. 4a, serotonin is significantly reduced in patients with diffuse SSc compared with patients with limited SSc and healthy control subjects $(p<0.05$, t test). We also observed increased levels of VWF, a biomarker of endothelial activation, in the serum of patients with SSc (Fig. 4b).

\section{Fibroblast proliferation and a-smooth muscle actin expression}

Tissue fibrosis in SSc results from an increased release of extracellular matrix from aberrantly activated fibroblasts [16]. Therefore, we evaluated the effect of platelet releasates in human fibroblast proliferation and $\alpha$-SMA expression. Human lung fibroblasts were cultured in 24-well plates in DMEM supplemented with $10 \% \mathrm{vol} / \mathrm{vol}$ SSc or healthy control platelet supernatant. Figure 5a shows that SSc platelet releasate supernatants induced stronger fibroblast proliferation than healthy control platelet releasate supernatants $(p<0.001$, ANOVA; $p<0.05, t$ test; control vs. SSc platelet groups). Also, we observed greater $\alpha$-SMA expression by fibroblasts cultured with SSc than by control platelet releasates $(p<0.05$, ANOVA).

\section{Discussion}

Platelets are best known for their role in hemostasis and thrombosis, but they also play important roles in inflammation, angiogenesis, and wound healing. Due to their small size and permanent circulation, they are in close proximity to and in continuous interaction with the endothelium. The sprouting of new vessels and the activation of fibroblasts stimulated by platelets within injured tissues probably contribute to prevention of hemorrhage and accelerated wound healing. However, when this remodeling response occurs with no regulation or in the absence of an injury stimulus, the integrity of the vessel and the tissue surrounding it can be impaired. Some of the first evidence 

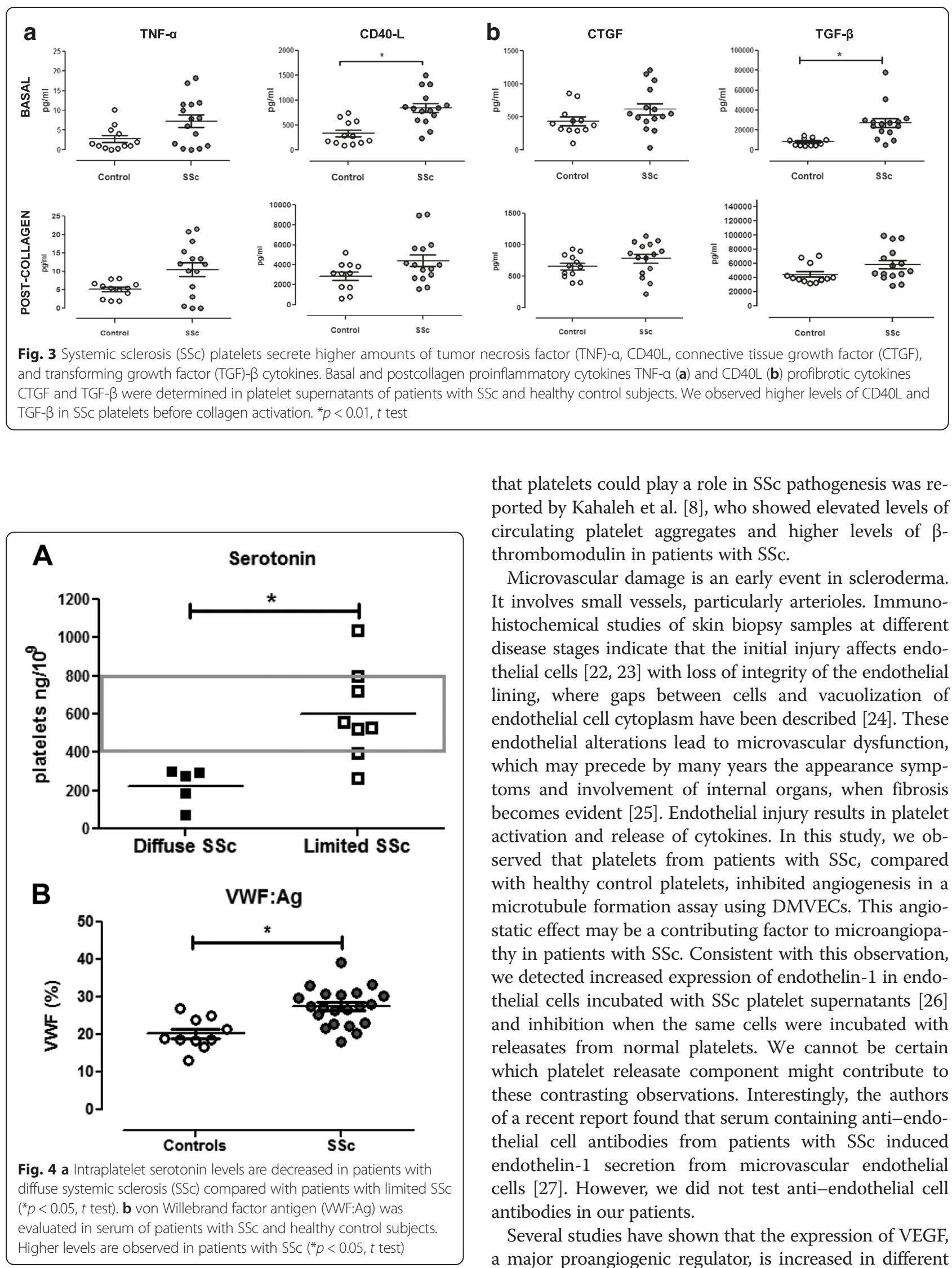

that platelets could play a role in SSc pathogenesis was reported by Kahaleh et al. [8], who showed elevated levels of circulating platelet aggregates and higher levels of $\beta$ thrombomodulin in patients with SSc.

Microvascular damage is an early event in scleroderma. It involves small vessels, particularly arterioles. Immunohistochemical studies of skin biopsy samples at different disease stages indicate that the initial injury affects endothelial cells $[22,23]$ with loss of integrity of the endothelial lining, where gaps between cells and vacuolization of endothelial cell cytoplasm have been described [24]. These endothelial alterations lead to microvascular dysfunction, which may precede by many years the appearance symptoms and involvement of internal organs, when fibrosis becomes evident [25]. Endothelial injury results in platelet activation and release of cytokines. In this study, we observed that platelets from patients with SSc, compared with healthy control platelets, inhibited angiogenesis in a microtubule formation assay using DMVECs. This angiostatic effect may be a contributing factor to microangiopathy in patients with SSc. Consistent with this observation, we detected increased expression of endothelin-1 in endothelial cells incubated with SSc platelet supernatants [26] and inhibition when the same cells were incubated with releasates from normal platelets. We cannot be certain which platelet releasate component might contribute to these contrasting observations. Interestingly, the authors of a recent report found that serum containing anti-endothelial cell antibodies from patients with SSc induced endothelin-1 secretion from microvascular endothelial cells [27]. However, we did not test anti-endothelial cell antibodies in our patients.

Several studies have shown that the expression of VEGF, a major proangiogenic regulator, is increased in different 


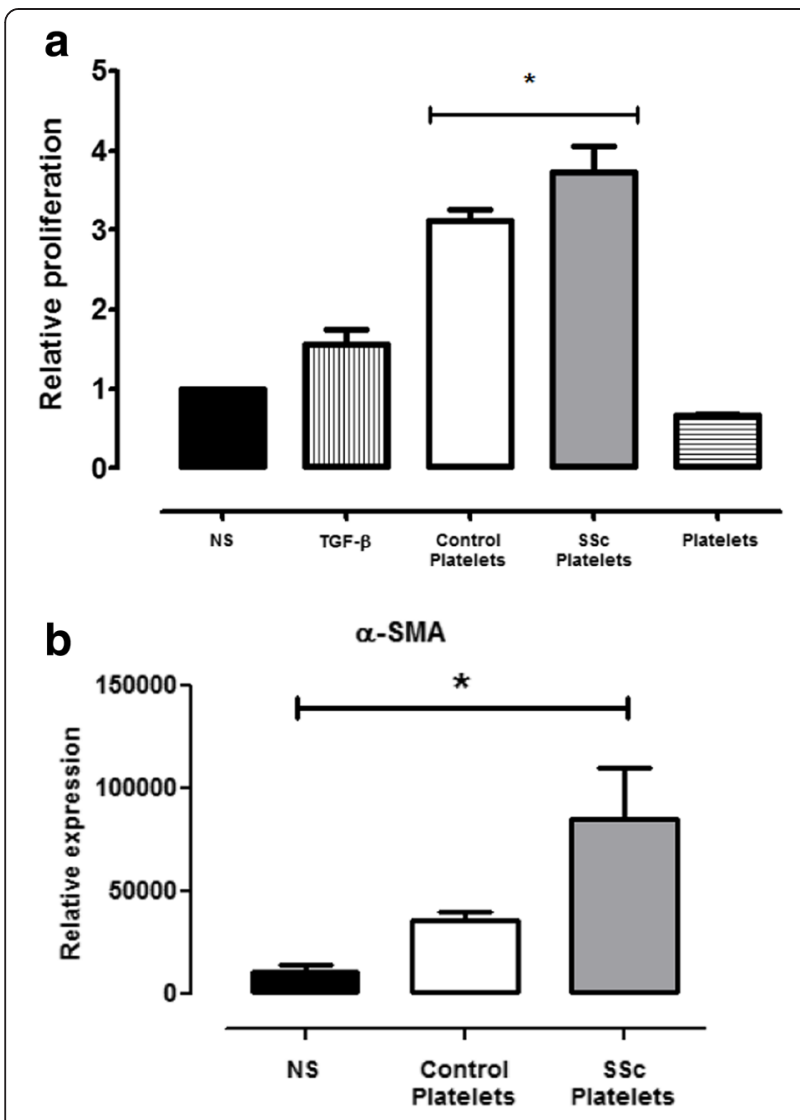

Fig. 5 Systemic sclerosis (SSc) platelet supernatant stimulates fibroblast proliferation and a-smooth muscle actin (a-SMA) expression. a Human fibroblast proliferation was evaluated using a 3-(4,5-dimethylthiazol-2-yl)2,5-diphenyltetrazolium bromide assay after $24 \mathrm{~h}$ culture alone [nonstimulated (NS)] or with transforming growth factor (TGF)- $\beta$ as well as with platelet releasates from patients with SSc and from healthy control subjects. The rightmost bar is a negative control and corresponds to normal platelets. Higher proliferation was observed after incubation with SSc platelet supernatants than with healthy control supernatants ( ${ }^{*} p<$ 0.05, Mann-Whitney $U$ test). $\mathbf{b}$ In the same experiment, a-SMA expression was evaluated in fibroblasts. Higher a-SMA expression was found in fibroblasts incubated with SSC platelet releasates than with healthy control releasates $\left({ }^{*} p<0.05\right.$, analysis of variance). Expression was determined by performing real-time polymerase chain reactions using TaqMan assays

cell types in the epidermis and dermis of these patients [18] and that the circulating levels of VEGF are also significantly increased in patients with SSc $[17,28]$. However, in SSc, angiogenesis is defective and the disease evolves toward a progressive loss of capillary vessels. Interestingly, the antiangiogenic splice variant $\mathrm{VEGF}_{165} \mathrm{~b}$ is upregulated in SSc skin and circulation [20,29], and it was suggested that profibrotic TGF- $\beta$ may contribute to the switch from proangiogenic to antiangiogenic VEGF isoforms [19]. The higher concentration of $\mathrm{VEGF}_{165} \mathrm{~b}$ in SSc platelet releasates that we observed, which is consistent with those previous reports, may contribute to angiogenesis inhibition and microangiopathy. It is well known that, among the more than 1000 proteins demonstrated in platelet releasates [30,31], platelets contain several angiogenesis regulatory proteins in their $\alpha$-granules. Angiogenesis is regulated by a highly sensitive interplay of growth factors and inhibitors, and their imbalance may lead to disease. In this regard, the increased ratio of antiangiogenic to angiogenic VEGF isoforms observed in these patients could be explained by increases in the antiangiogenic splice variant $\operatorname{VEGF}_{165} \mathrm{~b}$. Furthermore, researchers in a recent study found that the levels of C-X$\mathrm{C}$ motif chemokine ligand 4/platelet factor 4 , which is platelet-specific, and antiangiogenic and profibrotic chemokines are increased in patients with SSc and correlate with the level of fibrosis as well as the occurrence and progression of pulmonary arterial hypertension [32].

Some of the assessed platelet cytokines were increased in SSc. CD40L, a transmembrane protein of the TNF family, is expressed not only by immune system cells but also by activated platelets [33]. It is stored in platelets and is externalized and secreted during platelet activation [34, 35]. We observed that CD40L secretion in platelets from patients with SSc was significantly increased compared with platelets of control subjects $(p<$ $0.01, t$ test). We also found that TGF- $\beta$, a growth factor released from activated platelets, was significantly higher in platelet releasates from patients than in those from control subjects. TGF- $\beta$ stimulates angiogenesis, smooth muscle cells, and connective tissue fibroblasts [36], promoting the synthesis of collagens and matrix components [37]. In this regard, platelets are the main source of these molecules in the circulation [38-40], and the authors of previous reports have described increased levels of serum CD40L in scleroderma patients [41] and increased serum TGF- $\beta$ in patients with Raynaud's phenomenon [10].

Platelets contain almost all of the circulating serotonin, a vasoactive monoamine that is released upon platelet activation and which has been found to be increased in the plasma of patients with SSc [42]. We observed that intraplatelet serotonin was decreased in patients with diffuse SSc compared with patients with limited SSc and healthy control subjects. Given that the serotonin content of platelets increases as platelets age in the circulation, this finding may be explained by a decreased platelet lifespan in the circulation with a consequent increased turnover in diffuse SSc [43]. However, this is also compatible with the notion of damaged endothelium-mediated platelet activation and accelerated removal of platelets from the circulation. In this regard, TGF- $\beta$ and serotonin have been observed to be involved in fibrosis generation [21].

Normally, under the influence of appropriate stimuli, fibroblasts or their progenitor cells synthesize collagens and other extracellular matrix glycoproteins; secrete growth factors, cytokines, and chemokines; express surface receptors for these ligands; and undergo transdifferentiation into myofibroblasts [44]. These are specialized 
cells that arise from fibroblasts in response to TGF- $\beta$ stimuli, which express the cytoskeletal protein $\alpha$-SMA and synthesize collagens, tissue inhibitors of metalloproteases, and other extracellular matrix components $[45,46]$. Several alterations in fibroblasts have been described in the pathogenesis of scleroderma [25]. Our results show that platelet releasates from patients with SSc stimulate human fibroblast proliferation and increase $\alpha$-SMA expression, further contributing to disease progression.

\section{Conclusions}

Platelets are involved in several forms of pathologic angiogenesis, tissue repair, and fibrosis. From this point of view, our results highlight the role of platelets in SSc pathogenesis. Our findings suggest that antiangiogenic factors such as $\mathrm{VEGF}_{165} \mathrm{~b}$, together with proinflammatory $(\mathrm{CD} 40 \mathrm{~L})$ and profibrotic (TGF- $\beta$ ) factors secreted by platelets, can contribute to the defective angiogenesis and vascular repair in SSc. A better understanding of how platelets transport and deliver angiogenic, inflammatory and fibrotic regulatory proteins, as well as their contribution to SSc development and perpetuation, may have novel therapeutic implications.

\begin{abstract}
Abbreviations
ANA: antinuclear antibodies; ANOVA: analysis of variance; ATCC: American Type Culture Collection; CDNA: complementary DNA; CRP: C-reactive protein; CTGF: connective tissue growth factor; DMEM: Dulbecco's modified Eagle's medium; DMVEC: dermal human microvascular endothelial cell; EBM-2: endothelial growth basal medium; ELISA: enzyme-linked immunosorbent assay; ESR: erythrocyte sedimentation rate; HPLC: highperformance liquid chromatography; IQR: interquartile range; mRNA: messenger RNA; MTT: 3-(4,5-dimethylthiazol-2-yl)-2,5-diphenyltetrazolium bromide; NS: non-stimulated; PCR: polymerase chain reaction; RT: Reverse transcription; SD: standard deviation; a-SMA: $\alpha$-smooth muscle actin; SSc: systemic sclerosis; TGF- $\beta$ : transforming growth factor $\beta$; TNF-a: tumor necrosis factor $a$; VEGF: vascular endothelial growth factor; VEGF 165 b: vascular endothelial growth factor, 165b isoform; WWF: von Willebrand factor; WBC: white blood cell count.
\end{abstract}

\section{Competing interests}

The authors declare that they have no competing interests.

\begin{abstract}
Authors' contributions
DH carried out platelet isolation, immunoassays, and molecular genetic studies and drafted the manuscript. PIB participated in the design of the study, statistical analysis and drafting of the manuscript. VM participated in the design of the study, patient evaluation, and drafting of the manuscript. JD participated in the design of the study and patient evaluation. MB participated in immunoassays. CGS participated in cell culture experiments and drafted the manuscript. OP carried out the serotonin and von Willebrand factor assays and drafted the manuscript. DM participated in the design of the study, data interpretation, and critical revision of the manuscript. Ml participated in study design and coordination, patient evaluation, platelet isolation and immunoassays, and writing the final version of the manuscript. All authors were involved in drafting the manuscript, revising it critically, and approved the final version.
\end{abstract}

\section{Acknowledgments}

This work was supported by National Fund for Scientific and Technological Development (FONDECYT) grants 11100013 and 1130853. DH is a National Commission for Scientific and Technological Research (CONICYT) fellow.

\section{Author details}

'Departamento de Inmunología Clínica y Reumatología, Pontificia Universidad Católica de Chile, Marcoleta 350, Santiago, Chile. ${ }^{2}$ Departamento de Hematología-Oncología, Facultad de Medicina, Pontificia Universidad Católica de Chile, Santiago, Chile.

Received: 18 May 2015 Accepted: 4 November 2015

Published online: 19 November 2015

\section{References}

1. Chifflot H, Fautrel B, Sordet C, Chatelus E, Sibilia J. Incidence and prevalence of systemic sclerosis: a systematic literature review. Semin Arthritis Rheum. 2008;37:223-35.

2. Coral-Alvarado P, Pardo AL, Castaño-Rodriguez N, Rojas-Villarraga A, Anaya JM. Systemic sclerosis: a world wide global analysis. Clin Rheumatol. 2009; 28:757-65.

3. Denton CP, Black CM. Scleroderma-clinical and pathological advances. Best Pract Res Clin Rheumatol. 2004;18:271-90

4. Abraham DJ, Varga J. Scleroderma: from cell and molecular mechanisms to disease models. Trends Immunol. 2005;26:587-95.

5. Gabrielli A, Svegliati S, Moroncini G, Avvedimento EV. Pathogenic autoantibodies in systemic sclerosis. Curr Opin Immunol. 2007;19:640-5.

6. Hunzelmann N, Brinckmann J. What are the new milestones in the pathogenesis of systemic sclerosis? Ann Rheum Dis. 2010;69 Suppl 1:52-6. 7. Leslie M. Beyond clotting: the powers of platelets. Science. 2010;328:562-4.

8. Kahaleh MB, Osborn I, Leroy EC. Elevated levels of circulating platelet aggregates and beta-thromboglobulin in scleroderma. Ann Intern Med. 1982;96:610-3.

9. Postlethwaite AE, Chiang TM. Platelet contributions to the pathogenesis of systemic sclerosis. Curr Opin Rheumatol. 2007;19:574-9.

10. Silveri F, De Angelis R, Poggi A, Muti S, Bonapace G, Argentati F, et al. Relative roles of endothelial cell damage and platelet activation in primary Raynaud's phenomenon (RP) and RP secondary to systemic sclerosis. Scand J Rheumatol. 2001:30:290-6.

11. Lonzetti LS, Joyal F, Raynauld JP, Roussin A, Goulet JR, Rich E, et al. Updating the American College of Rheumatology preliminary classification criteria for systemic sclerosis: addition of severe nailfold capillaroscopy abnormalities markedly increases the sensitivity for limited scleroderma. Arthritis Rheum. 2001;44:735-6.

12. LeRoy EC, Medsger Jr TA. Criteria for the classification of early systemic sclerosis. J Rheumatol. 2001;28:1573-6.

13. Panes O, Matus V, Sáez CG, Quiroga T, Pereira J, Mezzano D. Human platelets synthesize and express functional tissue factor. Blood. 2007;109:5242-50.

14. Quiroga T, Goycoolea M, Belmont S, Panes O, Aranda E, Zúníiga P, et al. Quantitative impact of using different criteria for the laboratory diagnosis of type 1 von Willebrand disease. J Thromb Haemost. 2014;12:1238-43.

15. Kumar AM, Kumar M, Deepika K, Fernandez JB, Eisdorfer C. A modified HPLC technique for simultaneous measurement of 5-hydroxytryptamine and 5-hydroxyindoleacetic acid in cerebrospinal fluid, platelet and plasma. Life Sci. 1990;47:1751-9.

16. Gabrielli A, Avvedimento EV, Krieg T. Scleroderma. N Engl J Med. 2009:360:1989-2003

17. Distler O, Del Rosso A, Giacomelli R, Cipriani P, Conforti ML, Guiducci S, et al. Angiogenic and angiostatic factors in systemic sclerosis: increased levels of vascular endothelial growth factor are a feature of the earliest disease stages and are associated with the absence of fingertip ulcers. Arthritis Res. 2002;4:R11.

18. Ioannou M, Pyrpasopoulou A, Simos G, Paraskeva E, Nikolaidou C, Venizelos I, et al. Upregulation of VEGF expression is associated with accumulation of HIF-1a in the skin of naive scleroderma patients. Mod Rheumatol. 2013;23:1245-8.

19. Bates DO, Mavrou A, Qiu Y, Carter JG, Hamdollah-Zadeh M, Barratt S, et al. Detection of VEGF-A $A_{x x x} b$ isoforms in human tissues. PLoS One. 2013;8, e68399.

20. Manetti M, Guiducci S, Romano E, Ceccarelli C, Bellando-Randone S, Conforti ML, et al. Overexpression of $\mathrm{VEGF}_{165}$ b, an inhibitory splice variant of vascular endothelial growth factor, leads to insufficient angiogenesis in patients with systemic sclerosis. Circ Res. 2011;109:e14-26.

21. Dees C, Akhmetshina A, Zerr P, Reich N, Palumbo K, Horn A, et al. Plateletderived serotonin links vascular disease and tissue fibrosis. J Exp Med. 2011; 208:961-72.

22. Prescott RJ, Freemont AJ, Jones CJ, Hoyland J, Fielding P. Sequential dermal microvascular and perivascular changes in the development of scleroderma. J Pathol. 1992;166:255-63.

23. Sgonc R, Gruschwitz MS, Boeck G, Sepp N, Gruber J, Wick G. Endothelial cell apoptosis in systemic sclerosis is induced by antibody-dependent cell-mediated cytotoxicity via CD95. Arthritis Rheum. 2000;43:2550-62. 
24. Kahaleh B. Vascular disease in scleroderma: mechanisms of vascular injury. Rheum Dis Clin North Am. 2008;34:57-71.

25. Varga J, Abraham D. Systemic sclerosis: a prototypic multisystem fibrotic disorder. J Clin Invest. 2007:117:557-67.

26. Shi-Wen X, Chen Y, Denton CP, Eastwood M, Renzoni EA, Bou-Gharios G, et al. Endothelin-1 promotes myofibroblast induction through the ETA receptor via a rac/phosphoinositide 3-kinase/Akt-dependent pathway and is essential for the enhanced contractile phenotype of fibrotic fibroblasts. Mol Biol Cell. 2004;15:2707-19.

27. Corallo C, Franci B, Lucani B, Montella A, Chirico C, Gonnelli S, et al. From microvasculature to fibroblasts: contribution of anti-endothelial cell antibodies in systemic sclerosis. Int J Immunopathol Pharmacol. 2015;28:93-103.

28. Papaioannou Al, Zakynthinos E, Kostikas K, Kiropoulos T, Koutsokera A, Ziogas A, et al. Serum VEGF levels are related to the presence of pulmonary arterial hypertension in systemic sclerosis. BMC Pulm Med. 2009;9:18.

29. Manetti M, Guiducci S, Romano E, Bellando-Randone S, Lepri G, Bruni C, et al. Increased plasma levels of the VEGF $_{165}$ b splice variant are associated with the severity of nailfold capillary loss in systemic sclerosis. Ann Rheum Dis. 2013;72:1425-7.

30. Fong KP, Barry C, Tran AN, Traxler EA, Wannemacher KM, Tang HY, et al. Deciphering the human platelet sheddome. Blood. 2011;117:e15-26.

31. Mumford AD, Frelinger 3rd AL, Gachet $C$, Gresele $P$, Noris $P$, Harrison $P$, et al A review of platelet secretion assays for the diagnosis of inherited platelet secretion disorders. Thromb Haemost. 2015;114:14-25.

32. van Bon L, Affandi AJ, Broen J, Christmann RB, Marijnissen RJ, Stawski L, et al. Proteome-wide analysis and CXCL4 as a biomarker in systemic sclerosis. N Engl J Med. 2014;370:433-43.

33. Chatzigeorgiou A, Lyberi M, Chatzilymperis G, Nezos A, Kamper E. CD40/ CD40L signaling and its implication in health and disease. Biofactors. 2009;35:474-83.

34. Henn V, Slupsky JR, Gräfe M, Anagnostopoulos I, Förster R, Müller-Berghaus $\mathrm{G}$, et al. CD40 ligand on activated platelets triggers an inflammatory reaction of endothelial cells. Nature. 1998;391:591-4.

35. Hermann A, Rauch BH, Braun M, Schrör K, Weber AA. Platelet CD40 ligand (CD40L) - subcellular localization, regulation of expression, and inhibition by clopidogrel. Platelets. 2001;12:74-82.

36. Nurden AT, Nurden P, Sanchez M, Andia I, Anitua E. Platelets and wound healing. Front Biosci. 2008;13:3532-48.

37. Anitua E, Andia I, Ardanza B, Nurden P, Nurden AT. Autologous platelets as a source of proteins for healing and tissue regeneration. Thromb Haemost. 2004;91:4-15.

38. Assoian RK, Komoriya A, Meyers CA, Miller DM, Sporn MB. Transforming growth factor- $\beta$ in human platelets. Identification of a major storage site, purification, and characterization. J Biol Chem. 1983;258:7155-60.

39. Cicha I, Garlichs CD, Daniel WG, Goppelt-Struebe M. Activated human platelets release connective tissue growth factor. Thromb Haemost. 2004;91:755-60.

40. Kubota S, Kawata K, Yanagita T, Doi H, Kitoh T, Takigawa M. Abundant retention and release of connective tissue growth factor (CTGF/CCN2) by platelets. J Biochem. 2004;136:279-82.

41. Komura K, Sato S, Hasegawa M, Fujimoto M, Takehara K. Elevated circulating CD40L concentrations in patients with systemic sclerosis. J Rheumatol. 2004;31:514-9.

42. Stachów A, Jabłońska S, Skiendzielewska A. Biogenic amines derived from tryptophan in systemic and cutaneous scleroderma. Acta Derm Venereol. 1979:59:1-5

43. Mezzano D, Aranda E, Rodríguez S, Foradori A, Lira P. Increase in density and accumulation of serotonin by human aging platelets. Am J Hematol. 1984:17:11-21.

44. Sorrell JM, Caplan Al. Fibroblasts-a diverse population at the center of it all. Int Rev Cell Mol Biol. 2009;276:161-214.

45. Abraham DJ, Eckes B, Rajkumar V, Krieg T. New developments in fibroblast and myofibroblast biology: implications for fibrosis and scleroderma. Curr Rheumatol Rep. 2007;9:136-43.

46. Liu ZJ, Zhuge Y, Velazquez OC. Trafficking and differentiation of mesenchymal stem cells. J Cell Biochem. 2009;106:984-91.

\section{Submit your next manuscript to BioMed Central and take full advantage of:}

- Convenient online submission

- Thorough peer review

- No space constraints or color figure charges

- Immediate publication on acceptance

- Inclusion in PubMed, CAS, Scopus and Google Scholar

- Research which is freely available for redistribution

Submit your manuscript at www.biomedcentral.com/submit 